\title{
The Numerical Viscosity of Entropy Stable Schemes for Systems of Conservation Laws. I*
}

\author{
By Eitan Tadmor**
}

\begin{abstract}
Discrete approximations to hyperbolic systems of conservation laws are studied. We quantify the amount of numerical viscosity present in such schemes, and relate it to their entropy stability by means of comparison. To this end, conservative schemes which are also entropy conservative are constructed. These entropy conservative schemes enjoy second-order accuracy; moreover, they can be interpreted as piecewise linear finite element methods, and hence can be formulated on various mesh configurations. We then show that conservative schemes are entropy stable, if and-for three-point schemes-only if they contain more viscosity than that present in the above-mentioned entropy conservative ones.
\end{abstract}

1. Introduction. The systems of conservation laws referred to in the title are of the form

$$
\frac{\partial}{\partial t} \mathbf{u}+\sum_{k=1}^{d} \frac{\partial}{\partial x_{k}}\left[\mathbf{f}^{(k)}\right]=0, \quad(\bar{x}, t) \in \mathbf{R}^{d} \times[0, \infty) ;
$$

here $\mathbf{f}^{(k)} \equiv \mathbf{f}^{(k)}(\mathbf{u})=\left(f_{1}^{(k)}, \ldots, f_{N}^{(k)}\right)^{T}$ are smooth nonlinear flux functions of the conservative variables $\mathbf{u} \equiv \mathbf{u}(\bar{x}, t)=\left(u_{1}, \ldots, u_{N}\right)^{T}$. Owing to the nonlinearity of the fluxes $\mathbf{f}^{(k)}$, solutions of (1.1) may develop singularities at a finite time, after which one must admit weak solutions, i.e., those derived directly from the underlying integral conservative relations.

Yet, such weak solutions of the conservative equations are not unique. Additional criteria are required in order to single out a unique physically relevant weak solution, the latter being identified as, roughly speaking, a stable limit of a vanishing viscosity mechanism. Entropy stability is then sought as the usual criterion to identify such vanishing viscosity solutions. Lax [10] has shown that entropy stability is in fact equivalent to a vanishing viscosity mechanism, at least in the small - in the large for scalar problems, e.g., [8].

Received September 30, 1985; revised July 7, 1986.

1980 Mathematics Subject Classification (1985 Revision). Primary 35L65, 65M10; Secondary 39A11. $39 \mathrm{~A} 12$.

*Research was supported in part by the National Aeronautics and Space Administration under NASA Contract No. NAS1-17070 while the author was in residence at ICASE, NASA Langley Research Center, Hampton, VA 23665-5225. Additional support was provided by NSF (Grant No. DMS85-03294 and ARO (irant No. DAAG29-85-K-0190 while in residence at the University of California, Los Angeles, CA $9(0) 24$.

**Bat-Sheva Foundation Fellow. 
We study entropy stable approximations to such systems of conservation laws. Here entropy stability manifests itself in terms of a conservative cell entropy inequality. We note in passing that, if it holds for a large enough class of entropy functions, such a cell entropy inequality is intimately related to both the question of convergence toward a limit solution as well as the question of this limit solution being the unique physically relevant one, e.g., [1], [4], [17] and the references therein. It should also be mentioned in this connection that there are different kinds of estimates, other than the cell entropy inequality, which guarantee convergence and uniqueness, e.g., [19].

Starting with von Neumann and Richtmyer [13], it has long been a common practice to ensure the entropy stability of conservative schemes by tuning their numerical viscosity. In this paper we quantify precisely how much numerical viscosity is to be added. As in [20], this is accomplished by means of comparison: We show that entropy stability is achieved, if and-for three-point schemes-only if there is more numerical viscosity than that present in certain entropy conservative schemes. To this end we proceed as follows.

In Section 2 we begin by discussing the entropy variables associated with systems of conservation laws. As observed by Mock [12], [5], such systems are symmetrized with respect to these variables. Such symmetrization provides us with a natural order which then fits our goal to compare the numerical viscosities of different schemes. Expressed in terms of these entropy variables, we then turn to construct the above-mentioned entropy conservative schemes. The entropy conservative schemes - discussed in Sections 3 and 4-are second-order accurate and can be interpreted as piecewise linear finite element methods. In Section 5 we compare the amounts of viscosity present in different conservative schemes. It is shown that conservative schemes containing more viscosity than that of an entropy stable scheme are also entropy stable. In particular, comparison with the previously discussed entropy conservative schemes leads us to a sufficient and - for three-point schemes-necessary criterion for entropy stability. Since our entropy conservative schemes are second-order accurate, such a comparison applies to first- as well as second-order accurate entropy stable schemes.

We remark that Osher [14] has previously characterized entropy stable schemes with the help of a certain cell entropy identity, which subsequently is used to verify entropy stability for a number of well-known, first- as well as second-order accurate schemes [14], [15], [16]. The novelty of our equivalent entropy stability characterization presented in Section 5 lies in the notion of order; this becomes transparent once the entropy variables - rather than the conservative ones-are used, and the entropy conservative finite element schemes are constructed. Specific examples will be demonstrated in a subsequent paper.

Finally, in order to simplify the presentation, we treat the one-dimensional case; the multidimensional extension can be worked out dimension by dimension.

2. The Entropy Variables. We begin our discussion with the one-dimensional model

$$
\frac{\partial}{\partial t} \mathbf{u}+\frac{\partial}{\partial x}[\mathbf{f}(\mathbf{u})]=0
$$


We assume that the system (2.1) is equipped with a generalized

Entropy Function: a convex function $U=U(\mathbf{u})$ augmented with an entropy flux function $F=F(\mathbf{u})$ such that the follo ving compatibility relation holds,

$$
U_{\mathbf{u}}^{T} A=F_{\mathbf{u}}^{T} \text {. }
$$

Here, $A=A(\mathbf{u})$ is the Jacobian matrix

$$
A(\mathbf{u})=\mathbf{f}_{\mathbf{u}} .
$$

We note that the entropy functions, $U(\mathbf{u})$, are exactly those whose positive Hessians $U_{\text {uu }}>0$ symmetrize the system (2.1) upon multiplication on the left [2], [21]:

$$
U_{\mathrm{uu}} A=\left[U_{\mathrm{uu}} A\right]^{T} \text {. }
$$

Mock [12], [5] has pointed out a more fundamental symmetrization of system (2.1), preserving both the strong as well as the weak solutions of the system. To this end one makes use of the entropy variables

$$
\mathbf{v} \equiv \mathbf{v}(\mathbf{u})=\frac{\partial U}{\partial \mathbf{u}}(\mathbf{u})
$$

Thanks to the convexity of $U(\mathbf{u})$, the mapping $\mathbf{u} \rightarrow \mathbf{v}$ is one-to-one. Hence we can make the change of variables $\mathbf{u}=\mathbf{u}(\mathbf{v})$ which puts the system (2.1) in its equivalent symmetric form

$$
\frac{\partial}{\partial t}[\mathbf{u}(\mathbf{v})]+\frac{\partial}{\partial x}[\mathbf{g}(\mathbf{v})]=0, \quad \mathbf{g}(\mathbf{v}) \equiv \mathbf{f}(\mathbf{u}(\mathbf{v})) .
$$

The system (2.5a) is symmetric in the sense that the Jacobians of its temporal and spatial fluxes are

$$
H \equiv H(\mathbf{v})=\frac{\partial}{\partial \mathbf{v}}[\mathbf{u}(\mathbf{v})]>0, \quad B \equiv B(\mathbf{v})=\frac{\partial}{\partial \mathbf{v}}[\mathbf{g}(\mathbf{v})] .
$$

This follows from the compatibility relation (2.2), equivalently expressed as

$$
\mathbf{v}^{T} B(\mathbf{v})=G_{\mathbf{v}}^{T}, \quad G(\mathbf{v}) \equiv F(\mathbf{u}(\mathbf{v}))
$$

which in turn implies

$$
\begin{array}{ll}
\mathbf{u}(\mathbf{v})=\frac{\partial \phi}{\partial \mathbf{v}}, & \phi \equiv \phi(\mathbf{v})=\left[\mathbf{v}^{T} \mathbf{u}(\mathbf{v})-U(\mathbf{u}(\mathbf{v}))\right], \\
\mathbf{g}(\mathbf{v})=\frac{\partial \psi}{\partial \mathbf{v}}, & \psi \equiv \psi(\mathbf{v})=\left[\mathbf{v}^{T} \mathbf{g}(\mathbf{v})-G(\mathbf{v})\right] .
\end{array}
$$

Indeed, the Jacobians $H(\mathbf{v})$ and $B(\mathbf{v})$ in $(2.5 b)$ are the symmetric Hessians of the corresponding expressions inside the brackets in the right of (2.7).

Finally, we note that in contrast to the symmetrization on the left, quoted in (2.3), the use of the entropy variables symmetrizes the system (2.1) on the right [18], i.e., the original Jacobian $A=\mathbf{f}_{\mathbf{u}}$ is replaced here by the symmetric one, $B=\mathbf{g}_{\mathbf{v}}$,

$$
B=A U_{\mathrm{uu}}^{-1}=\left[A U_{\mathrm{uu}}^{-1}\right]^{T} \text {. }
$$

3. Entropy Stable Schemes. We consider conservative discretizations of the form $* * *$

$$
\frac{d}{d t} \mathbf{u}_{\nu}(t)=-\frac{1}{\Delta x}\left[\mathbf{f}_{\nu+1 / 2}-\mathbf{f}_{\nu-1 / 2}\right]
$$

\footnotetext{
***Both the differential and the discrete formulas will employ the same notations. The distinction between the two is made by the use of Greek indices in the discrete formulation.
} 
serving as consistent approximations to the system of conservation laws

$$
\frac{\partial}{\partial t} \mathbf{u}+\frac{\partial}{\partial x}[\mathbf{f}(\mathbf{u})]=0
$$

Here, $\mathbf{u}_{\nu}(t)$ denotes the approximation value along the gridline $\left(x_{\nu} \equiv \nu \Delta x, t\right), \Delta x$ being the spatial mesh size, and

$$
\mathbf{f}_{\nu+1 / 2}=\phi_{\mathbf{f}}\left(\mathbf{u}_{\nu-p+1}, \ldots, \mathbf{u}_{\nu+p}\right)
$$

is the Lipschitz continuous numerical flux consistent with the differential one,

$$
\phi_{\mathbf{f}}(\mathbf{w}, \mathbf{w}, \ldots, \mathbf{w})=\mathbf{f}(\mathbf{w}) .
$$

To discuss entropy stability, we let $(U, F)$ be a given entropy pair associated with the system (3.2). Multiplying by $U_{u}^{T}$ and employing (2.2), we conclude that under the smooth regime we have the additional conservation of entropy

$$
\frac{\partial U}{\partial t}+\frac{\partial F}{\partial x}=0
$$

Taking into account the nonsmooth regime as well, following Lax [10] and Kružkov [8] we postulate as an admissibility criterion an entropy stability requirement, expressed in terms of the following

Entropy Inequality: We have, in the sense of distributions,

$$
\frac{\partial U}{\partial t}+\frac{\partial F}{\partial x} \leqslant 0
$$

Similarly, for the scheme (3.1) to be entropy stable, a discrete cell entropy inequality is sought [4]:

$$
\frac{d}{d t} U\left(\mathbf{u}_{\nu}(t)\right)+\frac{1}{\Delta x}\left[F_{\nu+1 / 2}-F_{\nu-1 / 2}\right] \leqslant 0
$$

here, $F_{v+1 / 2}$ is the numerical entropy flux

$$
F_{\nu+1 / 2}=\Phi_{F}\left(\mathbf{u}_{\nu-p+1}, \ldots, \mathbf{u}_{\nu+p}\right)
$$

consistent with the differential one,

$$
\Phi_{F}(\mathbf{w}, \mathbf{w}, \ldots, \mathbf{w})=F(\mathbf{w}) .
$$

In order to examine the entropy stability question, we first study the case in which equality holds in (3.6a), i.e., we study entropy conservative schemes.

4. Entropy Conservative Schemes. In this section we identify particular schemes which satisfy a cell entropy equality. The numerical viscosity present in such entropy conservative schemes will then be used as the building block for the identification of entropy stable schemes.

In order to carry out the program above, it will prove useful to work with the entropy variables rather than the usual conservative ones. Thus, associated with an entropy function $U(\mathbf{u})$ are the entropy variables $\mathbf{v}=U_{\mathbf{u}}(\mathbf{u})$. Expressed in terms of the latter, the system of conservation laws considered is, see (2.5a),

$$
\frac{\partial}{\partial t}[\mathbf{u}(\mathbf{v})]+\frac{\partial}{\partial x}[\mathbf{g}(\mathbf{v})]=0, \quad \mathbf{g}(\mathbf{v})=\mathbf{f}(\mathbf{u}(\mathbf{v})) .
$$


It is augmented by the corresponding entropy inequality

$$
\frac{\partial V}{\partial t}+\frac{\partial G}{\partial x} \leqslant 0
$$

where $(V, G)$ is the appropriate entropy pair

$$
V \equiv V(\mathbf{v})=U(\mathbf{u}(\mathbf{v})), \quad G \equiv G(\mathbf{v})=F(\mathbf{u}(\mathbf{v})) .
$$

In a similar manner, we interpret the conservative scheme (3.1) in terms of the appropriate entropy variables $\mathbf{v}_{v}=U_{\mathbf{u}}\left(\mathbf{u}_{\nu}\right)$. Thus, (4.1) is approximated by

$$
\frac{d}{d t}\left[\mathbf{u}\left(\mathbf{v}_{\nu}(t)\right)\right]=-\frac{1}{\Delta x}\left[\mathbf{g}_{\nu+1 / 2}-\mathbf{g}_{\nu-1 / 2}\right]
$$

where $\mathbf{g}_{\nu+1 / 2}$ is the numerical flux

$$
\mathbf{g}_{\nu+1 / 2}=\psi_{\mathbf{g}}\left(\mathbf{v}_{\nu-p+1}, \ldots, \mathbf{v}_{\nu+p}\right), \quad \psi_{\mathbf{g}}\left(\cdots \mathbf{v}_{\nu} \cdots\right) \equiv \phi_{\mathbf{f}}\left(\cdots \mathbf{u}\left(\mathbf{v}_{\nu}\right) \cdots\right)
$$

consistent with the differential one,

$$
\psi_{\mathbf{g}}(\mathbf{w}, \mathbf{w}, \ldots, \mathbf{w})=\mathbf{g}(\mathbf{w}) .
$$

The corresponding cell entropy inequality takes the form

$$
\frac{d}{d t} V\left(\mathbf{v}_{\nu}(t)\right)+\frac{1}{\Delta x}\left[G_{\nu+1 / 2}-G_{\nu-1 / 2}\right] \leqslant 0 .
$$

Here, $G_{v+1 / 2}$ is the numerical entropy flux

$$
G_{\nu+1 / 2}=\Psi_{G}\left(\mathbf{v}_{\nu-p+1}, \ldots, \mathbf{v}_{\nu+p}\right)
$$

consistent with the differential one,

$$
\Psi_{G}(\mathbf{w}, \mathbf{w}, \ldots, \mathbf{w})=G(\mathbf{w}) .
$$

We seek entropy conservative schemes, i.e., schemes for which equality holds in (4.4a). To this end we multiply the scheme (4.3a) on the left by $\mathbf{v}_{v}^{T}(t) \equiv U_{\mathbf{u}}^{T}\left(\mathbf{u}_{\nu}(t)\right)$, obtaining

$$
\frac{d}{d t} V\left(\mathbf{v}_{\nu}(t)\right) \equiv \frac{d}{d t} U\left(\mathbf{u}_{\nu}(t)\right)=-\frac{1}{\Delta x} \mathbf{v}_{\nu}^{T}\left[\mathbf{g}_{\nu+1 / 2}-\mathbf{g}_{\nu-1 / 2}\right] .
$$

It follows that the expression on the right is conservative, i.e.,

$$
\mathbf{v}_{\nu}^{T}\left[\mathbf{g}_{\nu+1 / 2}-\mathbf{g}_{\nu-1 / 2}\right]=G_{\nu+1 / 2}-G_{\nu-1 / 2},
$$

if and only if the expression $\left[\mathbf{v}_{\nu+1}-\mathbf{v}_{\nu}\right]^{T} \mathbf{g}_{\nu+1 / 2}$ is, namely, if and only if there exists a grid function $\psi_{\nu}$ such that

$$
\Delta \mathbf{v}_{\nu+1 / 2}^{T} \mathbf{g}_{\nu+1 / 2}=\psi_{\nu+1}-\psi_{\nu}, \quad \Delta \mathbf{v}_{\nu+1 / 2} \equiv \mathbf{v}_{\nu+1}-\mathbf{v}_{\nu} .
$$

Indeed, in view of the identity

$$
\begin{aligned}
\mathbf{v}_{\nu}^{T}\left[\mathbf{g}_{\nu+1 / 2}-\mathbf{g}_{\nu-1 / 2}\right] \equiv & \frac{1}{2}\left[\mathbf{v}_{\nu}+\mathbf{v}_{\nu+1}\right]^{T} \mathbf{g}_{\nu+1 / 2}-\frac{1}{2}\left[\mathbf{v}_{\nu-1}+\mathbf{v}_{\nu}\right]^{T} \mathbf{g}_{\nu-1 / 2} \\
& -\left[\frac{1}{2}\left[\mathbf{v}_{\nu+1}-\mathbf{v}_{\nu}\right]^{T} \mathbf{g}_{\nu+1 / 2}+\frac{1}{2}\left[\mathbf{v}_{\nu}-\mathbf{v}_{\nu-1}\right]^{T} \mathbf{g}_{\nu-1 / 2}\right]
\end{aligned}
$$

the equality (4.5b) implies (4.5a) with

$$
G_{\nu+1 / 2}=\frac{1}{2}\left[\mathbf{v}_{\nu}+\mathbf{v}_{\nu+1}\right]^{T} \mathbf{g}_{\nu+1 / 2}-\frac{1}{2}\left(\psi_{\nu}+\psi_{\nu+1}\right) .
$$


Hence, schemes which fulfill (4.5b) are entropy conservative, i.e., we have

$$
\frac{d}{d t} V\left(\mathbf{v}_{\nu}(t)\right)+\frac{1}{\Delta x}\left[G_{\nu+1 / 2}-G_{\nu-1 / 2}\right]=0 .
$$

Our entropy conservative schemes fulfilling (4.5b) will be determined by setting the numerical flux $\mathbf{g}_{\nu+1 / 2}$ to be

$$
\mathbf{g}_{\nu+1 / 2}=\mathbf{g}_{\nu+1 / 2}^{*} \equiv \int_{\xi=0}^{1} \mathbf{g}\left(\mathbf{v}_{\nu+1 / 2}(\xi)\right) d \xi
$$

where the following abbreviations are used:

$$
\mathbf{v}_{\nu+1 / 2}(\xi) \equiv \mathbf{v}_{\nu}+\xi \Delta \mathbf{v}_{\nu+1 / 2}, \quad \Delta \mathbf{v}_{\nu+1 / 2} \equiv \mathbf{v}_{\nu+1}-\mathbf{v}_{\nu} .
$$

With this choice of numerical flux we have

$$
\Delta \mathbf{v}_{\nu+1 / 2}^{T} \mathbf{g}_{\nu+1 / 2}^{*}=\int_{\xi=0}^{1} \Delta \mathbf{v}_{\nu+1 / 2}^{T} \mathbf{g}\left(\mathbf{v}_{\nu}+\xi \Delta \mathbf{v}_{\nu+1 / 2}\right) d \xi .
$$

It is here where we make use of the entropy variables formulation: Thanks to the symmetry of $\mathbf{g}_{\mathrm{v}}$, the expression on the right equals the path-independent integral, see $(2.7 b)$,

$$
\begin{aligned}
\Delta \mathbf{v}_{\nu+1 / 2}^{T} \mathbf{g}_{\nu+1 / 2}^{*} & =\int_{\xi=0}^{1} \Delta \mathbf{v}_{\nu+1 / 2}^{T} \mathbf{g}\left(\mathbf{v}_{\nu}+\xi \Delta \mathbf{v}_{\nu+1 / 2}\right) d \xi \\
& =\int_{\mathbf{v}_{\nu}}^{\mathbf{v}_{\nu+1}} d \mathbf{v}^{T} \mathbf{g}(\mathbf{v})=\psi\left(\mathbf{v}_{\nu+1}\right)-\psi\left(\mathbf{v}_{\nu}\right),
\end{aligned}
$$

and therefore the conservation requirement $(4.5 b)$ is fulfilled in this case with $\psi_{\nu}=\psi\left(\mathbf{v}_{\nu}\right)$.

Let us summarize what we have shown in (2.7), (4.5) and (4.8) by stating

THEOREM 4.1 (Entropy conservative schemes). The conservative scheme

$$
\frac{d}{d t}\left[\mathbf{u}\left(\mathbf{v}_{\nu}(t)\right)\right]=-\frac{1}{\Delta x}\left[\mathbf{g}_{\nu+1 / 2}^{*}-\mathbf{g}_{\nu-1 / 2}^{*}\right], \quad \mathbf{g}_{\nu+1 / 2}^{*} \equiv \int_{\xi=0}^{1} \mathbf{g}\left(\mathbf{v}_{\nu+1 / 2}(\xi)\right) d \xi
$$

is also entropy conservative, i.e., it satisfies the following cell entropy equality

$$
\frac{d}{d t} V\left(\mathbf{v}_{\nu}(t)\right)+\frac{1}{\Delta x}\left[G_{\nu+1 / 2}^{*}-. G_{\nu-1 / 2}^{*}\right]=0
$$

Here, $G_{v+1 / 2}^{*}$ is the consistent numerical entropy flux given by

$$
\begin{aligned}
G_{\nu+1 / 2}^{*}= & \frac{1}{2}\left[\mathbf{v}_{\nu}+\mathbf{v}_{\nu+1}\right]^{T} \mathbf{g}_{\nu+1 / 2}^{*}-\frac{1}{2}\left[\psi\left(\mathbf{v}_{\nu}\right)+\psi\left(\mathbf{v}_{\nu+1}\right)\right] \\
= & \frac{1}{2}\left[\mathbf{v}_{\nu}+\mathbf{v}_{\nu+1}\right]^{T} \mathbf{g}_{\nu+1 / 2}^{*}+\frac{1}{2}\left[G\left(\mathbf{v}_{\nu}\right)+G\left(\mathbf{v}_{\nu+1}\right)\right] \\
& -\frac{1}{2}\left[\mathbf{v}_{\nu}^{T} \mathbf{g}\left(\mathbf{v}_{\nu}\right)+\mathbf{v}_{\nu+1}^{T} \mathbf{g}\left(\mathbf{v}_{\nu+1}\right)\right] .
\end{aligned}
$$

Remark. Another way of deriving the entropy conservation of scheme (4.9) follows from Osher's formula [14, Section 3]. Expressed in terms of the entropy variables, $\mathbf{v}_{\nu}$, Osher's formula asserts that for any conservative scheme of the form (4.3) the following identity holds,

$$
\frac{d}{d t} V\left(\mathbf{v}_{\nu}(t)\right)+\frac{1}{\Delta x}\left[G_{\nu+1 / 2}-G_{\nu-1 / 2}\right] \equiv \frac{1}{\Delta x} \int_{\mathbf{v}_{\nu}}^{\mathbf{v}_{\nu+1}} d \mathbf{v}^{T}\left[\mathbf{g}_{\nu+1 / 2}-\mathbf{g}(\mathbf{v})\right] .
$$


Hence, in view of our choice $\mathbf{g}_{v+1 / 2}=\mathbf{g}_{v+1 / 2}^{*}$ in (4.8), the (path-independent) integral on the right-hand side vanishes in this case, and entropy conservation follows.

We close this section by noting that the scheme (4.9), besides being entropy conservative, is also second-order accurate. Indeed, by the trapezoidal rule we have

$$
\mathbf{g}_{\nu+1 / 2}^{*}=\int_{\xi=0}^{1} \mathbf{g}\left(\mathbf{v}_{\nu+1 / 2}(\xi)\right) d \xi=\frac{1}{2}\left[\mathbf{g}\left(\mathbf{v}_{\nu}\right)+\mathbf{g}\left(\mathbf{v}_{\nu+1}\right)\right]+O\left(\left|\Delta \mathbf{v}_{\nu+1 / 2}\right|^{2}\right),
$$

and hence, for smooth functions, the right-hand side of (4.9) amounts to the usual second-order accurate central differencing modulo $O(\Delta x)^{2}$ local truncation error,

$$
-\frac{1}{\Delta x}\left[\mathbf{g}_{\nu+1 / 2}^{*}-\mathbf{g}_{\nu-1 / 2}^{*}\right]=-\frac{1}{2 \Delta x}\left[\mathbf{f}\left(\mathbf{u}_{\nu+1}\right)-\mathbf{f}\left(\mathbf{u}_{\nu-1}\right)\right]+O(\Delta x)^{2} .
$$

To shed a different light on this conclusion of second-order accuracy, we now demonstrate that the entropy conservative scheme (4.9) can be interpreted as a piecewise linear finite element method. Such a finite element formulation was extensively studied by Hughes and his collaborators, cf. [7].

Since we are interested in the semidiscrete case, we turn to consider the spatially weak formulation of (4.1),

$$
\int_{\Omega} \omega^{T} \frac{\partial}{\partial t}[\mathbf{u}(\mathbf{v})] d x d t=\int_{\Omega} \frac{\partial \omega^{T}}{\partial x} \mathbf{g}(\mathbf{v}) d x d t .
$$

Let the trial solutions $\mathbf{v} \rightarrow \hat{\mathbf{v}}(x, t) \equiv \sum_{k_{k}} \mathbf{v}_{k}(t) \hat{H}_{k}(x)$ and the weighting test functions $\omega \rightarrow \hat{\omega}(x, t) \equiv \sum_{k} \omega_{k}(t) \hat{H}_{k}(x)$ be chosen out of the typical finite element set spanned by the $C^{0}$ "hat functions"

$$
\hat{H}_{k}(x)= \begin{cases}\frac{x-x_{k-1}}{x_{k}-x_{k-1}}, & x_{k-1} \leqslant x \leqslant x_{k}, \\ \frac{x_{k+1}-x}{x_{k+1}-x_{k}}, & x_{k} \leqslant x \leqslant x_{k+1} .\end{cases}
$$

The spatial part on the right of (4.11) yields-after change of variables-our entropy conservative differencing

$$
\begin{aligned}
& \int_{x_{\nu-1}}^{x_{\nu+1}} \frac{\partial \hat{H}_{\nu}(x)}{\partial x} \mathbf{g}\left[\hat{\mathbf{v}}(x, t)=\sum_{\nu-1}^{\nu+1} \mathbf{v}_{k}(t) \hat{H}_{k}(x)\right] d x \\
&=-\left[\int_{\xi=0}^{1} \mathbf{g}\left(\mathbf{v}_{\nu+1 / 2}(\xi)\right) d \xi-\int_{\xi=0}^{1} \mathbf{g}\left(\mathbf{v}_{\nu-1 / 2}(\xi)\right) d \xi\right] .
\end{aligned}
$$

A second-order accurate mass lumping of the temporal term on the left of (4.11) leads to

$$
\begin{aligned}
\left.\int_{x_{\nu-1}}^{x_{\nu+1}} \hat{H}_{\nu}(x) \frac{\partial}{\partial t} \mathbf{u} \hat{\mathbf{v}}(x, t)=\sum_{\nu-1}^{\nu+1} \mathbf{v}_{k}(t) \hat{H}_{k}(x)\right] d x \\
=\frac{x_{\nu}-x_{\nu-1}}{2}\left[\frac{\partial}{\partial t} \mathbf{u}\left(\mathbf{v}_{\nu}(t)\right)+O\left(\left|\Delta \mathbf{v}_{\nu-1 / 2}\right|^{2}\right)\right] \\
\quad+\frac{x_{\nu+1}-x_{\nu}}{2}\left[\frac{\partial}{\partial t} \mathbf{u}\left(\mathbf{v}_{\nu}(t)\right)+O\left(\left|\Delta \mathbf{v}_{\nu+1 / 2}\right|^{2}\right)\right] .
\end{aligned}
$$


Equating (4.12a) and (4.12b), and neglecting the quadratic error terms, we arrive at

$$
\begin{aligned}
\frac{\partial}{\partial t}\left[\mathbf{u}\left(\mathbf{v}_{\nu}(t)\right)\right]=\frac{-2}{x_{\nu+1}-x_{\nu-1}} & {\left[\int_{\xi=0}^{1} \mathbf{g}\left(\mathbf{v}_{\nu+1 / 2}(\xi)\right) d \xi\right.} \\
& \left.-\int_{\xi=0}^{1} \mathbf{g}\left(\mathbf{v}_{\nu-1 / 2}(\xi)\right) d \xi\right] .
\end{aligned}
$$

Observe that (4.13) also suggests the natural extension of the entropy conservative schemes (4.9) to nonequally spaced meshes. Finally, we point out that a global entropy conservation for the unlumped version of (4.11) follows at once by selecting the test functions $\hat{\omega}(x, t)$ to coincide with the trial function $\hat{\mathbf{v}}$, so that in view of (2.6), Eq. (4.11) yields

$$
0=\int_{\Omega}\left[\hat{\mathbf{v}}^{T} \frac{\partial}{\partial t}[\mathbf{u}(\hat{\mathbf{v}})]-\frac{\partial \hat{\mathbf{v}}^{T}}{\partial x} \mathbf{g}(\hat{\mathbf{v}})\right] d x d t \equiv \int_{\Omega}\left[\frac{\partial}{\partial t} V(\hat{\mathbf{v}})+\frac{\partial}{\partial x} G(\hat{\mathbf{v}})\right] d x d t
$$

The mass lumping in (4.12b), which results in the explicit scheme (4.13), enables the localization of the entropy conservation to each cell.

5. Numerical Viscosity and Entropy Stability. The essential role played by the numerical viscosity has long been recognized, starting with von Neumann and Richtmyer [13]. In this section we quantify the amount of numerical viscosity required in order to guarantee entropy stability. As in [20], this will be done by means of comparison.

To begin with, we consider the entropy conservative scheme (4.9). Integration by parts of its numerical flux formula (4.6a) yields

$$
\begin{aligned}
\mathbf{g}_{\nu+1 / 2}^{*}= & \int_{\xi=0}^{1} \mathbf{g}\left(\mathbf{v}_{\nu+1 / 2}(\xi)\right) d \xi=\left.\left(\xi-\frac{1}{2}\right) \mathbf{g}\left(\mathbf{v}_{\nu+1 / 2}(\xi)\right)\right|_{\xi=0} ^{\xi=1} \\
& -\int_{\xi=0}^{1}\left(\xi-\frac{1}{2}\right) \frac{d}{d \xi} \mathbf{g}\left(\mathbf{v}_{\nu+1 / 2}(\xi)\right) d \xi
\end{aligned}
$$

Recalling the notation for the Jacobian $\mathbf{g}_{\mathbf{v}}$, see $(2.5 b)$,

$$
B(\mathbf{v})=\mathbf{g}_{\mathbf{v}},
$$

the right-hand side of $(5.1)$ can be rewritten as

$$
\mathbf{g}_{\nu+1 / 2}^{*}=\frac{1}{2}\left[\mathbf{g}\left(\mathbf{v}_{\nu}\right)+\mathbf{g}\left(\mathbf{v}_{\nu+1}\right)\right]-\frac{1}{2} \int_{\xi=0}^{1}(2 \xi-1) B\left(\mathbf{v}_{\nu+1 / 2}(\xi)\right) d \xi \Delta \mathbf{v}_{\nu+1 / 2} .
$$

Inserting this into (4.9), our entropy conservative scheme assumes the viscosity form

$$
\begin{aligned}
\frac{d}{d t}\left[\mathbf{u}\left(\mathbf{v}_{\nu}(t)\right)\right]= & -\frac{1}{2 \Delta x}\left[\mathbf{g}\left(\mathbf{v}_{\nu+1}\right)-\mathbf{g}\left(\mathbf{v}_{\nu-1}\right)\right] \\
& +\frac{1}{2 \Delta x}\left[Q_{\nu+1 / 2}^{*} \Delta \mathbf{v}_{\nu+1 / 2}-Q_{\nu-1 / 2}^{*} \Delta \mathbf{v}_{\nu-1 / 2}\right] .
\end{aligned}
$$

Here, $Q_{\nu+1 / 2}^{*}$ is the numerical viscosity coefficient matrix given by

$$
Q_{\nu+1 / 2}^{*}=\int_{\xi=0}^{1}(2 \xi-1) B\left(\mathbf{v}_{\nu+1 / 2}(\xi)\right) d \xi
$$

We note that the second brackets on the right of (5.3a) mimic a diffusive-like term $\Delta x\left(Q^{*} \mathbf{v}_{x}\right)_{x}$. Yet, though the viscosity matrix $Q_{\nu+1 / 2}^{*}$ is symmetric, it is not necessarily a positive definite one; rather, it is determined so as to counterbalance the dispersive flux central differencing inside the first brackets on the right of (5.3a). 
Motivated by the discussion above, we would like to consider schemes given in a similar viscosity form [20]

$$
\begin{aligned}
\frac{d}{d t}\left[\mathbf{u}\left(\mathbf{v}_{\nu}(t)\right)\right]= & -\frac{1}{2 \Delta x}\left[\mathbf{g}\left(\mathbf{v}_{\nu+1}\right)-\mathbf{g}\left(\mathbf{v}_{\nu-1}\right)\right] \\
& +\frac{1}{2 \Delta x}\left[Q_{\nu+1 / 2} \Delta \mathbf{v}_{\nu+1 / 2}-Q_{\nu-1 / 2} \Delta \mathbf{v}_{\nu-1 / 2}\right] .
\end{aligned}
$$

The matrix $Q_{\nu+1 / 2}$ on the right will be referred to as the numerical viscosity coefficient matrix.

Remark. We observe that the above definition of the numerical viscosity coefficient depends on the specific entropy function under consideration, $U(\mathbf{u})$. The special choice $U(u)=\frac{1}{2} u^{2}$ corresponds to our earlier viscosity definition [20] in the scalar case.

Which schemes admit a viscosity form like (5.4)? To answer this question, we observe that the numerical flux determined by (5.4),

$$
\begin{aligned}
\mathbf{g}_{\nu+1 / 2} & =\psi_{\mathbf{g}}\left(\mathbf{v}_{\nu-p+1}, \ldots, \mathbf{v}_{\nu+p}\right) \\
& =\frac{1}{2}\left[\mathbf{g}\left(\mathbf{v}_{\nu}\right)+\mathbf{g}\left(\mathbf{v}_{\nu+1}\right)\right]-\frac{1}{2} Q_{\nu+1 / 2}\left[\mathbf{v}_{\nu+1}-\mathbf{v}_{\nu}\right],
\end{aligned}
$$

satisfies the consistency relation

$$
\psi_{\mathbf{g}}\left(\mathbf{v}_{\nu-p+1}, \ldots, \mathbf{v}_{\nu-1}, \mathbf{w}, \mathbf{w}, \mathbf{v}_{\nu+2}, \ldots, \mathbf{v}_{\nu+p}\right)=\mathbf{g}(\mathbf{w}) .
$$

The consistency relation (5.6) is slightly more stringent than the usual one in (4.3c). It characterizes the class of essentially three-point schemes, a class which includes, beside the standard three-point schemes, most of the recently constructed secondorder accurate TVD schemes, e.g., [3], [16], [6]. In the Appendix we show that the converse of the above implication holds, namely we have

LEMMA 5.1. The consistent conservative scheme (4.3) can be written in the viscous form (5.4) if and only if it is an essentially three-point scheme.

Granted the viscosity form (5.4), we now turn to discuss the question of entropy stability. We say that one scheme contains more viscosity than another scheme if the viscosity coefficient of the first scheme, say $Q_{\nu+1 / 2}^{(1)}$, dominates that of the second one, $Q_{\nu+1 / 2}^{(2)}$, i.e., if we have

$$
\Delta \mathbf{v}_{\nu+1 / 2}^{T} Q_{\nu+1 / 2}^{(2)} \Delta \mathbf{v}_{\nu+1 / 2} \leqslant \Delta \mathbf{v}_{\nu+1 / 2}^{T} Q_{\nu+1 / 2}^{(1)} \Delta \mathbf{v}_{\nu+1 / 2}
$$

A strengthened formulation of this in terms of the natural order among symmetric matrices is

$$
\operatorname{Re} Q_{\nu+1 / 2}^{(2)} \leqslant \operatorname{Re} Q_{\nu+1 / 2}^{(1)} .
$$

In case the entropy variables were used to begin with, they would lead to symmetric viscosity coefficients very much the same way as they led to symmetric Jacobians, e.g., (5.3b), and we would arrive at the natural hierarchy

$$
Q_{\nu+1 / 2}^{(2)} \leqslant Q_{\nu+1 / 2}^{(1)} \text {. }
$$

Remark. The last three inequalities are equivalent when dealing with scalar equations. Otherwise, the quadratic-like inequality (5.7a) is in fact-due to the dependence of $Q_{\nu+1 / 2}=Q\left(\ldots, \mathbf{v}_{\nu}, \mathbf{v}_{\nu+1}, \ldots\right)$ on $\Delta \mathbf{v}_{\nu+1 / 2}$-nonlinear; hence, the in- 
equalities (5.7b) and (5.7c) may serve as sufficient (but not necessary) criteria for verifying the entropy stability by comparison, as outlined below.

Equipped with the above terminology, we now turn to a particular comparison with the entropy conservative scheme (5.3). Thus, we consider a conservative scheme in its viscosity form (5.4) and let

$$
D_{\nu+1 / 2}=Q_{\nu+1 / 2}-Q_{\nu+1 / 2}^{*}
$$

denote the deviation of its viscosity from that of the entropy conservative scheme (5.3). The scheme considered in (5.4) then takes the form

$$
\begin{aligned}
\frac{d}{d t}\left[\mathbf{u}\left(\mathbf{v}_{\nu}(t)\right)\right]= & -\frac{1}{\Delta x}\left[\mathbf{g}_{\nu+1 / 2}^{*}-\mathbf{g}_{\nu+1 / 2}^{*}\right] \\
& +\frac{1}{2 \Delta x}\left[D_{\nu+1 / 2} \Delta \mathbf{v}_{\nu+1 / 2}-D_{\nu-1 / 2} \Delta \mathbf{v}_{\nu-1 / 2}\right] .
\end{aligned}
$$

Multiplying the last equality by $U_{\mathbf{u}}^{T}\left(\mathbf{u}_{\nu}(t)\right)=\mathbf{v}_{\nu}^{T}(t)$ on the left yields

$$
\begin{aligned}
& \frac{d}{d t} V\left(\mathbf{v}_{\nu}(t)\right)+\frac{1}{\Delta x} \mathbf{v}_{\nu}^{T}\left[\mathbf{g}_{\nu+1 / 2}^{*}-\mathbf{g}_{\nu-1 / 2}^{*}\right] \\
& \quad=\frac{1}{2 \Delta x} \mathbf{v}_{\nu}^{T}\left[D_{\nu+1 / 2} \Delta \mathbf{v}_{\nu+1 / 2}-D_{\nu-1 / 2} \Delta \mathbf{v}_{\nu-1 / 2}\right] .
\end{aligned}
$$

By Theorem 4.1 the second expression on the left-hand side of (5.10) equals the conservative difference

$$
\begin{aligned}
\frac{1}{\Delta x} \mathbf{v}_{\nu}^{T}\left[\mathbf{g}_{\nu+1 / 2}^{*}-\mathbf{g}_{\nu-1 / 2}^{*}\right] & =-\mathbf{v}_{\nu}^{T}(t) \frac{d}{d t}\left[\mathbf{u}\left(\mathbf{v}_{\nu}(t)\right)\right] \\
& =-\frac{d}{d t} V\left(\mathbf{v}_{\nu}(t)\right)=\frac{1}{\Delta x}\left[G_{\nu+1 / 2}^{*}-G_{\nu-1 / 2}^{*}\right] .
\end{aligned}
$$

Regarding the right-hand side of (5.10), the following identity puts it as the sum of familiar quadratic terms plus a conservative difference:

$$
\begin{aligned}
\frac{1}{2 \Delta x} \mathbf{v}_{\nu}^{T} & {\left[D_{\nu+1 / 2} \Delta \mathbf{v}_{\nu+1 / 2}-D_{\nu-1 / 2} \Delta \mathbf{v}_{\nu-1 / 2}\right] } \\
\equiv & -\frac{1}{2 \Delta x}\left[\frac{1}{2} \Delta \mathbf{v}_{\nu+1 / 2}^{T} D_{\nu+1 / 2} \Delta \mathbf{v}_{\nu+1 / 2}+\frac{1}{2} \Delta \mathbf{v}_{\nu-1 / 2}^{T} D_{\nu-1 / 2} \Delta \mathbf{v}_{\nu-1 / 2}\right] \\
& +\frac{1}{2 \Delta x}\left[\frac{1}{2}\left[\mathbf{v}_{\nu}+\mathbf{v}_{\nu+1}\right]^{T} D_{\nu+1 / 2} \Delta \mathbf{v}_{\nu+1 / 2}-\frac{1}{2}\left[\mathbf{v}_{\nu-1}+\mathbf{v}_{\nu}\right]^{T} D_{\nu-1 / 2} \Delta \mathbf{v}_{\nu-1 / 2}\right] .
\end{aligned}
$$

Using the last equality, we conclude the main result of this section, namely

THEOREM 5.2 (Entropy stability). (i) A conservative scheme which contains more numerical viscosity than that present in the entropy conservative one is entropy stable. Moreover, the entropy dissipates in this case at a rate governed by the cell estimate

$$
\begin{aligned}
& \frac{d}{d t} V\left(\mathbf{v}_{\nu}(t)\right)+\frac{1}{\Delta x}\left[G_{\nu+1 / 2}-G_{\nu-1 / 2}\right] \\
& \quad=-\frac{1}{4 \Delta x}\left[\Delta \mathbf{v}_{\nu+1 / 2}^{T} D_{\nu+1 / 2} \Delta \mathbf{v}_{\nu+1 / 2}+\Delta \mathbf{v}_{\nu-1 / 2}^{T} D_{\nu-1 / 2} \Delta \mathbf{v}_{\nu-1 / 2}\right] \leqslant 0 .
\end{aligned}
$$

Here, $G_{\nu+1 / 2}=\Psi_{G}\left(\mathbf{v}_{\nu}, \mathbf{v}_{\nu+1}\right)$ is the consistent numerical entropy flux

$$
G_{\nu+1 / 2}=G_{\nu+1 / 2}^{*}-\frac{1}{4}\left[\mathbf{v}_{\nu}+\mathbf{v}_{\nu+1}\right]^{T} D_{\nu+1 / 2} \Delta \mathbf{v}_{\nu+1 / 2}
$$


In the case of three-point schemes the converse also holds, namely,

(ii) A three-point conservative scheme which is entropy stable contains more numerical viscosity than that present in the entropy conservative one.

Proof. Inserting (5.11) and (5.12) into (5.10), we obtain

$$
\begin{aligned}
& \frac{d}{d t} V\left(\mathbf{v}_{\nu}(t)\right)+\frac{1}{\Delta x}\left[G_{\nu+1 / 2}-G_{\nu-1 / 2}\right] \\
& \quad=-\frac{1}{4 \Delta x}\left[\Delta \mathbf{v}_{\nu+1 / 2}^{T} D_{\nu+1 / 2} \Delta \mathbf{v}_{\nu+1 / 2}+\Delta \mathbf{v}_{\nu-1 / 2}^{T} D_{\nu-1 / 2} \Delta \mathbf{v}_{\nu-1 / 2}\right],
\end{aligned}
$$

where $G_{v+1 / 2}$ is the consistent numerical entropy flux given in (5.13b). Now, if the scheme considered contains more viscosity than the entropy conservative scheme, then by (5.8) the right-hand side of (5.14) is nonpositive, and entropy stability follows. To prove the converse, we borrow from the arguments used in [17, Theorem 7.3]. To this end, assume that our three-point scheme is entropy stable, i.e., that it satisfies a consistent cell entropy inequality of the form

$$
\frac{d}{d t} V\left(\mathbf{v}_{\nu}(t)\right)+\frac{1}{\Delta x}\left[\tilde{G}_{\nu+1 / 2}-\tilde{G}_{\nu-1 / 2}\right] \leqslant 0, \quad \tilde{G}_{\nu+1 / 2}=\tilde{\Psi}_{G}\left(\mathbf{v}_{\nu}, \mathbf{v}_{\nu+1}\right) .
$$

Subtracting this from (5.14), we get after mutliplication by $\Delta x$

$$
\text { (5.15a) } \quad H_{\nu+1 / 2}-H_{\nu-1 / 2} \geqslant-\frac{1}{4}\left[\Delta \mathbf{v}_{\nu+1 / 2}^{T} D_{\nu+1 / 2} \Delta \mathbf{v}_{\nu+1 / 2}+\Delta \mathbf{v}_{\nu-1 / 2}^{T} D_{\nu-1 / 2} \Delta \mathbf{v}_{\nu-1 / 2}\right] \text {. }
$$

Here, $H_{\nu+1 / 2}=H\left(\mathbf{v}_{\nu}, \mathbf{v}_{\nu+1}\right)$ stands for the difference between the two-point entropy fluxes, $G_{\nu+1 / 2}-\tilde{G}_{\nu+1 / 2}$, and hence satisfies the consistency relation, see $(4.4 \mathrm{~b}-\mathrm{c})$,

$$
H(\mathbf{w}, \mathbf{w})=0 .
$$

Choosing $\mathbf{v}_{\nu-1}=\mathbf{v}_{\nu}$ in (5.15a), we obtain, in view of (5.15b),

$$
H_{\nu+1 / 2} \geqslant-\frac{1}{4} \Delta \mathbf{v}_{\nu+1 / 2}^{T} D_{\nu+1 / 2} \Delta \mathbf{v}_{\nu+1 / 2}
$$

Similarly, taking $\mathbf{v}_{\nu}=\mathbf{v}_{\nu+1}$ in (5.15a) yields, in view of (5.15b),

$$
-H_{\nu-1 / 2} \geqslant-\frac{1}{4} \Delta \mathbf{v}_{\nu-1 / 2}^{T} D_{\nu-1 / 2} \Delta \mathbf{v}_{\nu-1 / 2} \text {. }
$$

Adding (5.16), together with (5.17) ${ }_{\nu+1}$ implies

$$
0=H_{\nu+1 / 2}-H_{\nu+1 / 2} \geqslant-\frac{1}{2} \Delta \mathbf{v}_{\nu+1 / 2} D_{\nu+1 / 2} \Delta \mathbf{v}_{\nu+1 / 2}
$$

or, according to (5.9),

$$
\Delta \mathbf{v}_{\nu+1 / 2}^{T} Q_{\nu+1 / 2}^{*} \Delta \mathbf{v}_{\nu+1 / 2} \leqslant \Delta \mathbf{v}_{\nu+1 / 2}^{T} Q_{\nu+1 / 2} \Delta \mathbf{v}_{\nu+1 / 2}
$$

Thus the scheme considered, (5.4), contains more viscosity than that present in the entropy conservative one, (5.3), as asserted.

We recall that the entropy conservative scheme (4.9) is second-order accurate. Hence, Theorem 5.2 allows us, in particular, to tune additional numerical viscosity so that we retain both the entropy stability and second-order accuracy [14]-[16].

Finally, using arguments similar to those employed in the proof of the last theorem, we conclude with the following extension of [20, Theorem 6.1], dealing with systems of conservation laws.

THEOREM 5.3 (Entropy stability by comparison). Conservative schemes containing more viscosity than an entropy stable scheme are also entropy stable.

Acknowledgment. I am grateful to Tom Hughes for a number of stimulating discussions and to Stanley Osher and Ed Harabetian for their comments. 


\section{APPENDIX}

A. The Viscosity Form of Essentially Three-Point Schemes. We consider conservative schemes in the viscous form

$$
\begin{aligned}
\frac{d}{d t}\left[\mathbf{u}\left(\mathbf{v}_{\nu}(t)\right)\right]= & \frac{1}{\Delta x}\left[\mathbf{g}_{\nu+1 / 2}-\mathbf{g}_{\nu-1 / 2}\right]=\frac{1}{2 \Delta x}\left[\mathbf{g}\left(\mathbf{v}_{\nu+1}\right)-\mathbf{g}\left(\mathbf{v}_{\nu-1}\right)\right] \\
& +\frac{1}{2 \Delta x}\left[Q_{\nu+1 / 2} \Delta \mathbf{v}_{\nu+1 / 2}-Q_{\nu-1 / 2} \Delta \mathbf{v}_{\nu-1 / 2}\right] .
\end{aligned}
$$

The numerical flux associated with scheme (a.1),

$$
\begin{aligned}
\mathbf{g}_{\nu+1 / 2} & =\psi_{\mathbf{g}}\left(\mathbf{v}_{\nu-p+1}, \ldots, \mathbf{v}_{\nu+p}\right) \\
& =\frac{1}{2}\left[\mathbf{g}\left(\mathbf{v}_{\nu}\right)+\mathbf{g}\left(\mathbf{v}_{\nu+1}\right)\right]-\frac{1}{2} Q_{\nu+1 / 2}\left[\mathbf{v}_{\nu+1}-\mathbf{v}_{\nu}\right],
\end{aligned}
$$

satisfies the essentially three-point consistency relation

$$
\psi_{\mathbf{g}}\left(\mathbf{v}_{\nu-p+1}, \ldots, \mathbf{v}_{\nu-1}, \mathbf{w}, \mathbf{w}, \mathbf{v}_{\nu+2}, \ldots, \mathbf{v}_{\nu+p}\right)=\mathbf{g}(\mathbf{w}) .
$$

Conversely, consider a conservative scheme

$$
\frac{d}{d t}\left[\mathbf{u}\left(\mathbf{v}_{\nu}(t)\right]=-\frac{1}{\Delta x}\left[\psi_{\mathbf{g}}\left(\mathbf{v}_{\nu-p+1}, \ldots, \mathbf{v}_{\nu+p}\right)-\psi_{\mathbf{g}}\left(\mathbf{v}_{\nu-p}, \ldots, \mathbf{v}_{\nu+p-1}\right)\right]\right.
$$

Subject to the essentially three-point consistency relation

$$
\psi_{\mathbf{g}}\left(\mathbf{v}_{\nu-p+1}, \ldots, \mathbf{v}_{\nu-1}, \mathbf{w}, \mathbf{w}, \mathbf{v}_{\nu+2}, \ldots, \mathbf{v}_{\nu+p}\right)=g(\mathbf{w}),
$$

the scheme (a.4) can be put into the viscosity form (a.1) provided a numerical viscosity coefficient, $Q_{\nu+1 / 2}$, can be found such that

$$
Q_{\nu+1 / 2} \Delta \mathbf{v}_{\nu+1 / 2}=\mathbf{g}\left(\mathbf{v}_{\nu}\right)+\mathbf{g}\left(\mathbf{v}_{\nu+1}\right)-2 \psi_{\mathbf{g}}\left(\mathbf{v}_{\nu-p+1}, \ldots, \mathbf{v}_{\nu+p}\right) .
$$

Using the consistency relation (a.5) we can rewrite the right-hand side of (a.6) as

$$
\begin{aligned}
& {\left[\psi_{\mathbf{g}}\left(\ldots, \mathbf{v}_{\nu}, \mathbf{v}_{\nu}, \ldots\right)-\psi_{\mathbf{g}}\left(\ldots \mathbf{v}_{\nu}, \mathbf{v}_{\nu+1}, \ldots\right)\right]} \\
& +\left[\psi_{\mathbf{g}}\left(\ldots, \mathbf{v}_{\nu+1}, \mathbf{v}_{\nu+1}, \ldots\right)-\psi_{\mathbf{g}}\left(\ldots, \mathbf{v}_{\nu}, \mathbf{v}_{\nu+1}, \ldots\right)\right],
\end{aligned}
$$

and equality (a.6) is then fulfilled by setting the numerical viscosity coefficient to be

$$
\begin{aligned}
Q_{\nu+1 / 2}= & -\int_{\xi=0}^{1} \frac{\partial}{\partial \mathbf{v}_{\nu+1}}\left[\psi_{\mathbf{g}}\left(\ldots, \mathbf{v}_{\nu}, \mathbf{v}_{\nu+1 / 2}(\xi), \ldots\right)\right] d \xi \\
& +\int_{\xi=0}^{1} \frac{\partial}{\partial \mathbf{v}_{\nu}}\left[\psi_{\mathbf{g}}\left(\ldots, \mathbf{v}_{\nu+1 / 2}(\xi), \mathbf{v}_{\nu+1} \ldots\right)\right] d \xi
\end{aligned}
$$

School of Mathematical Sciences

Tel-Aviv University

Tel-Aviv 69978, Israel

Institute for Computer Applications

in Science and Engineering

NASA Langley Research Center

Hampton, Virginia 23665-5225

1. R. J. DiPerna, "Convergence of approximate solutions to conservation laws," Arch. Rational Mech. Anal., v. 82, 1983, pp. 27-70.

2. K. O. Friedrichs \& P. D. Lax, "Systems of conservation laws with a convex extension," Proc. Nat. Acad. Sci. U.S.A., v. 68, 1971, pp. 1686-1688.

3. A. Harten, "High resolution schemes for hyperbolic conservation laws," J. Comput. Phys., v. 49, 1983. pp. 357-393. 
4. A. Hartin, J. M. Hyman \& P. D. Lax, “On finite difference approximations and entropy conditions for shocks," Comm. Pure Appl. Math., v. 29, 1976, pp. 297-322.

5. A. HaRTen \& P. D. LaX, "A random choice finite difference scheme for hyperbolic conservation laws," SIAM J. Numer. Anal., v. 18, 1981, pp. 289-315.

6. A. HARTEN \& S. OSHER, Uniformly High-Order Accurate Non-Oscillatory Schemes, I, MRC Technical Summary Report No. 2823, May 1985.

7. T. J. R. Hughes, M. Mallet \& L. P. Franca, "Entropy-stable finite element methods for compressible fluids; application to high mach number flows with shocks," Finite Element Methods for Nonlinear Problems, Springer-Verlag. (To appear.)

8. S. N. KRUŽKOV, "First-order quasilinear equations in several independent variables," Math. USSR-Sh., v. 10, 1970, pp. 217-243.

9. P. D. Lax, "Hyperbolic systems of conservation laws, II," Comm. Pure Appl. Math., v. 10, 1957. pp. 537-566.

10. P. D. Lax, Hyperbolic Systems of Conservation Laws and the Mathematical Theory of Shock Waves, SIAM Regional Conference Lecturers in Applied Mathematics, No. 11, 1972.

11. M. S. Mocк, A Difference Scheme Employing Fourth-Order Viscosity to Enforce an Entropy Inequality, Proc. Bat-Sheva Conference, Tel-Aviv University, 1977.

12. M. S. Mock. "Systems of conservation laws of mixed type," J. Differential Equations, v. 37, 1980. pp. $70-88$.

13. J. von NeUmanN \& R. D. RichtMyer, “A method for the numerical calculation of hydrodynamic shocks,” J. Appl. Phys., v. 21, 1950, pp. 232-237.

14. S. OShER, "Riemann solvers, the entropy condition, and difference approximations," SIAM J. Numer. Anal., v. 21, 1984, pp. 217-235.

15. S. OShER, "Convergence of generalized MUSCL schemes," SIAM J. Numer. Anal., v. 22, 1984, pp. 947-961.

16. S. Osher \& S. Chakravarthy, "High resolution schemes and the entropy condition," SIaM J. Numer. Anal., v. 21, 1984, pp. 955-984.

17. S. OSher \& E. TADMOR, The Convergence of Difference Approximations to Scalar Conservation Laws, ICASE Report No. 85-28, NASA CR-172614.

18. E. TAdmOR, "Skew-selfadjoint form for systems of cońservation laws," J. Math. Anal. Appl., v. 103,1984 , pp. $428-442$.

19. E. TADMOR, "The large-time behavior of the scalar, genuinely nonlinear Lax-Friedrichs scheme," Math. Comp., v. 43, 1984, pp. 353-368.

20. E. TADMOR, "Numerical viscosity and the entropy condition for conservative difference schemes," Math. Comp., v. 43, 1984, pp. 369-381.

21. E. TADMOR, "Entropy functions for symmetric systems of conservation laws," J. Math. Anal. Appl., v. 121, 1987. 